\title{
Про моногенні функцї на розширеннях комутативної алгебри
}

\author{
В. С. Шпаківський
}

Abstract. A relation between spatial potential fields and analytic functions
given in commutative algebras was established by P. W. Ketchum who has
shown that every analytic function $\Phi(\zeta)$ of the variable $\zeta=x e_{1}+y e_{2}+z e_{3}$ satisfies the equation

$$
\Delta_{3} u(x, y, z):=\left(\frac{\partial^{2}}{\partial x^{2}}+\frac{\partial^{2}}{\partial y^{2}}+\frac{\partial^{2}}{\partial z^{2}}\right) u(x, y, z)=0
$$

in the case where the elements $e_{1}, e_{2}, e_{3}$ of a commutative algebra satisfy the condition

$$
e_{1}^{2}+e_{2}^{2}+e_{3}^{2}=0
$$

because

$$
\frac{\partial^{2} \Phi}{\partial x^{2}}+\frac{\partial^{2} \Phi}{\partial y^{2}}+\frac{\partial^{2} \Phi}{\partial z^{2}} \equiv \Phi^{\prime \prime}(\zeta)\left(e_{1}^{2}+e_{2}^{2}+e_{3}^{2}\right)=0
$$

where $\Phi^{\prime \prime}:=\left(\Phi^{\prime}\right)^{\prime}$ and $\Phi^{\prime}(\zeta)$ is defined by the equality $d \Phi=\Phi^{\prime}(\zeta) d \zeta$.

I. P. Mel'nichenko noticed that doubly differentiable in the sense of Gateaux functions form the largest algebra of functions $\Phi$ satisfying identically the above equalities, where $\Phi^{\prime \prime}$ is the Gateaux second derivative of function $\Phi$.

It is proved in [1] that for constructing solutions of the equation

$$
\sum_{\alpha+\beta+\gamma=N} C_{\alpha, \beta, \gamma} \frac{\partial^{N} U}{\partial x^{\alpha} \partial y^{\beta} \partial z^{\gamma}}=0, \quad C_{\alpha, \beta, \gamma} \in \mathbb{R} .
$$

in the form of components of monogenic functions with values in finitedimensional commutative associative algebras it suffices to confine itself to studying monogenic functions in algebras of a certain type, that is, in algebras $\mathbb{A}_{n}$.

For $n$-dimensional $(2 \leq n<\infty)$ commutative associative algebra $\mathbb{A}_{n}$ we introduce a concept of expansion as a set of $(n+1)$-dimensional commutative associative algebras with certain multiplication rules. A relation between monogenic (continuous and differentiable in the sense of Gâteaux) functions

Робота виконана при підтримці Міністерства освіти і науки України (проект № 0116U001528).

2010 Mathematics Subject Classification: 30G35, 57R35

Ключові слова: commutative associative algebra, monogenic function, expansion of algebra 
in the algebra $\mathbb{A}_{n}$ and monogenic functions that defined on expansions of $\mathbb{A}_{n}$ is established. For the equation above it will mean the following: if its complex-valued solution $U(x, y, z)$ is a component of monogenic function in the algebra $\mathbb{A}_{n}$ for $n<N$, then there exists the algebra of the form $\mathbb{A}_{N}$ and there exists a monogenic function $\Phi$ in $\mathbb{A}_{N}$ such that $U(x, y, z)$ is a component of the function $\Phi$. The results obtained in this paper hold for the equations of the above type of $d$ variables for all integer $2 \leq d \leq 2 N$.

Анотація. Для $n$-вимірної $(2 \leq n<\infty)$ комутативної асоціативної алгебри $\mathbb{A}_{n}$ введено поняття розширення як сімейства $(n+1)$-вимірних комутативних асоціативних алгебр з певними правилами множення. Встановлено зв'язок між моногенними (неперервними і диференційовними за Гато) функціями в алгебрі $\mathbb{A}_{n}$ і моногенними функціями, визначеними на розширеннях алгебри $\mathbb{A}_{n}$.

\section{1. ПОСТАНОВКА ПРОБЛЕМИ}

Ідея про побудову розв'язків заданих диференціальних рівнянь в частинних похідних у вигляді компонент аналітичних функцій в комутативних алгебрах знаходить свій початок ще в роботі П. Кетчума [3]. Він використав аналітичні функції зі значення в комутативній алгебрі для побудови розв'язків тривимірного рівняння Лапласа.

Узагальнюючи П. Кетчума, М. Рошкулець [7], [8] використовував аналітичні функції зі значеннями в комутативних алгебрах для дослідження рівнянь вигляду

$$
\mathcal{L}_{N} U(x, y, z):=\sum_{\alpha+\beta+\gamma=N} C_{\alpha, \beta, \gamma} \frac{\partial^{N} U}{\partial x^{\alpha} \partial y^{\beta} \partial z^{\gamma}}=0, \quad C_{\alpha, \beta, \gamma} \in \mathbb{R} .
$$

Розглядаючи змінну $\zeta=x e_{1}+y e_{2}+z e_{3}$ i аналітичну функцію $\Phi(\zeta)$, отримуємо наступну рівність для мішаної похідної:

$$
\frac{\partial^{\alpha+\beta+\gamma} \Phi}{\partial x^{\alpha} \partial y^{\beta} \partial z^{\gamma}}=e_{1}^{\alpha} e_{2}^{\beta} e_{3}^{\gamma} \Phi^{(\alpha+\beta+\gamma)}(\zeta)=e_{1}^{\alpha} e_{2}^{\beta} e_{3}^{\gamma} \Phi^{(N)}(\zeta) .
$$

Підставляючи (1.2) в рівняння (1.1), маємо рівність

$$
\mathcal{L}_{N} \Phi(\zeta)=\Phi^{(N)}(\zeta) \sum_{\alpha+\beta+\gamma=N} C_{\alpha, \beta, \gamma} e_{1}^{\alpha} e_{2}^{\beta} e_{3}^{\gamma},
$$

яка показує, що для виконання рівності $\mathcal{L}_{N} \Phi(\zeta)=0$ елементи алгебри $e_{1}=1, e_{2}, e_{3}$ мають задовольняти таке характеристичне рівняння:

$$
\sum_{\alpha+\beta+\gamma=N} C_{\alpha, \beta, \gamma} e_{2}^{\beta} e_{3}^{\gamma}=0
$$


Якщо ліву частину рівняння (1.3) розкласти за базисом алгебри, то характеристичне рівняння (1.3) рівносильне характеристичній системі рівнянь, породженій рівнянням (1.3).

Таким чином, при виконанні умови (1.3) кожна аналітична функція $\Phi$ зі значеннями в довільній комутативній асоціативній алгебрі задовольняє рівняння (1.1), i, відповідно, усі дійснозначні компоненти функцї $\Phi$ є розв'язками рівняння (1.1).

В роботі [6] розглядаються диференціальні рівняння в частинних похідних від декількох змінних і наведено ряд прикладів на застосування описаного вище методу.

I. Мельниченко [4] запропонував розглядати в рівності (1.2) функції $\Phi$, що $N$ разів диференційовні за Гато. При цьому неперервну і диференційовну за Гато функцію I. Мельниченко назвав моногенною.

Підкреслимо, що описаний вище підхід до побудови розв'язків певних класів рівнянь з частинними похідними розвивається 3 використанням комутативних алгебр. I тому результати, що одержуються у цьому напрямку ніяк не можуть бути наслідками деяких схожих за формую результатів з кліффордового аналізу. Зокрема, в роботі [9] отримано конструктивний опис моногенних функцій (пов'язаних з рівнянням (1.1)) зі значеннями в довільній комутативній асоціативній алгебрі над полем $\mathbb{C}$ за допомогою голоморфних функцій комплексної змінної.

В роботі [11] показано, що для побудови розв'язків рівняння (1.1) у вигляді компонент моногенних функцій зі значеннями в скінченновимірних комутативних асоціативних алгебрах достатньо обмежитись вивченням моногенних функцій у алгебрах певного виду. Зупинимось детальніше на цьому результаті.

Нехай $\mathbb{N}$ - множина натуральних чисел і $m, n \in \mathbb{N}$ такі, що $m \leq n$. Нехай далі $\mathbb{A}_{n}^{m}$ - довільна комутативна асоціативна алгебра з одиницею над полем комплексних чисел $\mathbb{C}$. Е. Картан $[1$, с. 33] довів, що в алгебрі $\mathbb{A}_{n}^{m}$ існує базис $\left\{I_{k}\right\}_{k=1}^{n}$, який задовольняє наступні правила множення:

- для всіх $r, s \in[1, m] \cap \mathbb{N}$

$$
I_{r} I_{s}= \begin{cases}0 & \text { при } r \neq s, \\ I_{r} & \text { при } r=s ;\end{cases}
$$

- для всіх $r, s \in[m+1, n] \cap \mathbb{N}$

$$
I_{r} I_{s}=\sum_{k=\max \{r, s\}+1}^{n} \Upsilon_{r, k}^{s} I_{k}
$$


- для кожного $s \in[m+1, n] \cap \mathbb{N}$ існує таке $u_{s} \in[1, m] \cap \mathbb{N}$, що для Bcix $r \in[1, m] \cap \mathbb{N}$

$$
I_{r} I_{s}= \begin{cases}0 & \text { при } r \neq u_{s}, \\ I_{s} & \text { при } r=u_{s} .\end{cases}
$$

Відмітимо, що перші $m$ базисних векторів $\left\{I_{u}\right\}_{u=1}^{m}$ є ідемпотентами і породжують напівпросту підалгебру $S$ алгебри $\mathbb{A}_{n}^{m}$. Очевидно також, що вектори $\left\{I_{r}\right\}_{r=m+1}^{n}$ породжують нільпотентну підалгебру $N$ цієї алгебри. Крім того, з правил множення алгебри $\mathbb{A}_{n}^{m}$ випливає, що $\mathbb{A}_{n}^{m} \in$ напівпрямою сумою $m$-вимірної напівпростої підалгебри $S$ і $(n-m)$ вимірної нільпотентної підалгебри $N$, тобто

$$
\mathbb{A}_{n}^{m}=S \oplus_{s} N \text {. }
$$

Теорема 5.1 роботи [11] стверджує, що для побудови розв'язків диференціального рівняння (1.1) у вигляді компонент моногенних функцій зі значеннями в комутативних асоціативних алгебрах, достатньо обмежитись вивченням моногенних функцій в алгебрах з базисом

$$
\left\{1, \eta_{1}, \eta_{2}, \ldots, \eta_{n}\right\},
$$

де $\eta_{1}, \eta_{2}, \ldots, \eta_{n}$ - нільпотенти, тобто в алгебрах виду

$$
\mathbb{A}_{n}:=\mathbb{A}_{n}^{1}=1 \oplus_{s} N
$$

Це означає, що кількість таких $n$-вимірних комутативних асоціативних алгебр з одиницею над полем $\mathbb{C}$, в яких потрібно вивчати моногенні функції, дорівнює кількості $(n-1)$-вимірних комутативних асоціативних нільпотентних алгебр над $\mathbb{C}$. Наведемо таблицю з даними про кількість таких алгебр (відносно посилань по кількість таких алгебр див. зауваження 5.3 в роботі [11]).

\begin{tabular}{|c|c|c|}
\hline$n$ & кількість алгебр виду $\mathbb{A}_{n}^{m}$ & кількість алгебр виду $\mathbb{A}_{n}$ \\
\hline \hline 2 & 2 & 1 \\
\hline 3 & 4 & 2 \\
\hline 4 & 9 & 4 \\
\hline 5 & 25 & 9 \\
\hline 6 & 53 & 25 \\
\hline$\geq 7$ & $\infty$ & $\infty$ \\
\hline
\end{tabular}

У даній роботі буде встановлено зв'язок між моногенними функціями, що визначені в алгебрах $\mathbb{A}_{n}$ та в спеціальних алгебрах виду $\mathbb{A}_{n+1}$ (які буде названо розширеннями) при всіх натуральних $n \geq 2$. Для рівняння (1.1) це означатиме таке: якщо комплекснозначний розв'язок $U(x, y, z)$ рівняння $(1.1)$ є деякою компонентою моногенної функції в 
деякій алгебрі $\mathbb{A}_{n}$ при $n<N$, то серед алгебр виду $\mathbb{A}_{N}$ існує алгебра $\mathbb{A}$ і існує моногенна функція $\Phi$ в $\mathbb{A}$ така, що $U(x, y, z)$ є деякою компонентою функції $\Phi$.

\section{2. ХАРАКТЕРИСТИЧНЕ РіВНЯННЯ В АЛГЕБРАХ $\mathbb{A}_{n}$}

Спершу покажемо, що у всіх алгебрах виду $\mathbb{A}_{n}$ характеристичне рівняння (1.3) завжди має розв'язки.

3 правил множення алгебри $\mathbb{A}_{n}^{m}$ випливає, що таблиця множення алгебри $\mathbb{A}_{n}$ має вигляд

$$
I_{r} I_{s}=\sum_{k=\max \{r, s\}+1}^{n} \Upsilon_{r, k}^{s} I_{k}, \quad \forall r, s \in\{1,2, \ldots, n-1\},
$$

тобто

\begin{tabular}{c||c|c|c|c|c|}
$\cdot$ & 1 & $I_{1}$ & $I_{2}$ & $\ldots$ & $I_{n-1}$ \\
\hline \hline 1 & 1 & $I_{1}$ & $I_{2}$ & $\ldots$ & $I_{n-1}$ \\
\hline$I_{1}$ & $I_{1}$ & $\sum_{k=2}^{n-1} \Upsilon_{1, k}^{1} I_{k}$ & $\sum_{k=3}^{n-1} \Upsilon_{2, k}^{1} I_{k}$ & $\ldots$ & 0 \\
\hline$I_{2}$ & $I_{2}$ & $\sum_{k=3}^{n-1} \Upsilon_{2, k}^{1} I_{k}$ & $\sum_{k=3}^{n-1} \Upsilon_{2, k}^{2} I_{k}$ & $\ldots$ & 0 \\
\hline$\vdots$ & $\vdots$ & $\vdots$ & $\vdots$ & $\ddots$ & $\vdots$ \\
\hline$I_{n-1}$ & $I_{n-1}$ & 0 & 0 & $\cdots$ & 0 \\
\hline
\end{tabular}

де структурні константи алгебри $\Upsilon_{r, k}^{s} \in \mathbb{C}$ такі як і в рівності (1.4). Покладемо $I_{0}:=1$.

Лема 2.1. Для довільного елемента $a:=\sum_{k=0}^{n-1} a_{k} I_{k}, a_{k} \in \mathbb{C}$, алгебри $\mathbb{A}_{n}$ i довілъного натурального $\beta$ справедлива рівність

$$
a^{\beta}=a_{0}^{\beta}+\sum_{k=1}^{n-1} P_{k}\left(a_{0}, a_{1}, \ldots, a_{k}\right) I_{k}
$$

де $P_{k}$ - однорідний поліном степеня $\beta$ своїх аргументів.

Доведення. Скористаємось методом математичної індукції по $\beta$. При $\beta=1$ твердження леми очевидне. Припустимо, що формула (2.3) справедлива при $\beta=s$ :

$$
a^{s}=a_{0}^{s}+\sum_{k=1}^{n-1} P_{k}\left(a_{0}, a_{1}, \ldots, a_{k}\right) I_{k}
$$


де $P_{k}$ - однорідний поліном степеня $s$. Користуючись таблицею множення алгебри $\mathbb{A}_{n}$, доведемо справедливість формули $(2.3)$ при $\beta=s+1$. Отже, маємо:

$$
\begin{aligned}
a^{s+1}= & a^{s} a=a_{0}^{s+1}+I_{1}\left(a_{0}^{s} a_{1}+P_{1} a_{0}\right)+I_{2}\left(a_{0}^{s} a_{2}+P_{1} a_{1} \Upsilon_{1,1}^{2}+a_{0} P_{2}\right)+\cdots \\
\cdots & +I_{n-1}\left[a_{0}^{s} a_{n-1}+P_{n-1} a_{0}+\Upsilon_{1, n-1}^{1} a_{1} P_{1}+\Upsilon_{2, n-1}^{1}\left(a_{2} P_{1}+a_{1} P_{2}\right)+\right. \\
& +\Upsilon_{2, n-1}^{2} a_{2} P_{2}+\Upsilon_{3, n-1}^{1}\left(a_{3} P_{1}+a_{1} P_{3}\right)+\Upsilon_{3, n-1}^{1}\left(a_{3} P_{1}+a_{1} P_{3}\right)+ \\
& \left.+\Upsilon_{3, n-1}^{2}\left(a_{3} P_{2}+a_{2} P_{3}\right) \cdots+\Upsilon_{n-1, n-1}^{n-2} a_{n-2} P_{n-2}\right]=: \\
= & : a_{0}^{s+1}+\sum_{k=1}^{n-1} \widetilde{P}_{k}\left(a_{0}, a_{1}, \ldots, a_{k}\right) I_{k},
\end{aligned}
$$

де $\widetilde{P}_{k}$ - однорідний поліном степеня $s+1$.

Повністю аналогічно до леми 2.1, з використанням таблиці множення $(2.2)$, доводиться така лема.

Лема 2.2. Поліноми $P_{k}$ з рівності (2.3) подаються у вигляді

$$
P_{k}\left(a_{0}, a_{1}, \ldots, a_{k}\right)=\beta a_{0}^{\beta-1} a_{k}+\widehat{P}_{k}\left(a_{0}, a_{1}, \ldots, a_{k-1}\right),
$$

де $\widehat{P}_{k}$-однорідний поліном степеня $\beta$.

Теорема 2.3. $B$ кожній алгебрі виду $\mathbb{A}_{n}$ характеристичне рівняння (1.3) має розв'язки.

Доведення. Нехай

$$
e_{1}=1, \quad e_{2}=\sum_{r=0}^{n-1} a_{r} I_{r}, \quad e_{3}=\sum_{r=0}^{n-1} b_{r} I_{r}
$$

де $a_{r}, b_{r} \in \mathbb{C}$.

З леми 2.1 випливають рівності

$$
e_{2}^{\beta}=a_{0}^{\beta}+\sum_{k=1}^{n-1} P_{k}\left(a_{0}, a_{1}, \ldots, a_{k}\right) I_{k}, \quad e_{3}^{\gamma}=b_{0}^{\gamma}+\sum_{k=1}^{n-1} Q_{k}\left(b_{0}, b_{1}, \ldots, b_{k}\right) I_{k},
$$

де $P_{k}, Q_{k}$ - однорідні поліноми степенів $\beta$ і $\gamma$, відповідно. Тоді для лівої частини рівняння (1.3) маємо

$$
\begin{gathered}
\sum_{\alpha+\beta+\gamma=N} C_{\alpha, \beta, \gamma} e_{2}^{\beta} e_{3}^{\gamma}=\sum_{\alpha+\beta+\gamma=N} C_{\alpha, \beta, \gamma} a_{0}^{\beta} b_{0}^{\gamma}+I_{1} R_{1}\left(b_{0}^{\gamma} P_{1}, a_{0}^{\beta} Q_{1}\right)+ \\
+I_{2} R_{2}\left(b_{0}^{\gamma} P_{2}, a_{0}^{\beta} Q_{2}, P_{1} Q_{1}\right)+I_{3} R_{3}\left(b_{0}^{\gamma} P_{3}, a_{0}^{\beta} Q_{3}, P_{1} Q_{1}, P_{1} Q_{2}, P_{2} Q_{1}\right)+\cdots \\
\ldots+I_{n-1} R_{n-1}\left(b_{0}^{\gamma} P_{n-1}, a_{0}^{\beta} Q_{n-1}, P_{i} Q_{j}, i, j=\{1,2, \ldots, n-2\}\right),
\end{gathered}
$$


де $R_{k}$ - лінійна функція своїх аргументів. Отже, при $k=1,2, \ldots, n-1$ функція $R_{k}$ є однорідним поліномом степеня $\beta+\gamma$ від аргументів $a_{0}, b_{0}$, $a_{1}, b_{1}, \ldots, a_{k}, b_{k}$.

Таким чином, характеристичне рівняння (1.3) рівносильне такій характеристичній системі рівнянь:

$$
\begin{gathered}
\sum_{\alpha+\beta+\gamma=N} C_{\alpha, \beta, \gamma} a_{0}^{\beta} b_{0}^{\gamma}=0, \\
R_{1}\left(a_{0}, b_{0}, a_{1}, b_{1}\right)=0 \\
\ldots \ldots \ldots \ldots \ldots \ldots, \ldots \ldots \\
R_{n-1}\left(a_{0}, b_{0}, a_{1}, b_{1}, \ldots, a_{n-1}, b_{n-1}\right)=0,
\end{gathered}
$$

де поліноми $R_{k}$ визначені вище.

Перше рівняння системи (2.4) є комплексним поліномом від двох змінних степеня $\beta+\gamma$. Зафіксуємо $a_{0} \neq 0$. Тоді за основною теоремою алгебри 3 першого рівняння системи (2.4) знаходимо $b_{0}$. Тепер $a_{0}, b_{0}$ визначені. Тоді за лемою 2.2 друге рівняння системи (2.4) є лінійним відносно невідомих $a_{1}, b_{1}$. Зафіксуємо $a_{1}$. Тоді з лінійного відносно $b_{1}$ рівняння $R_{1}\left(a_{0}, b_{0}, a_{1}, b_{1}\right)=0$ знаходимо $b_{1}$ через $a_{0}, a_{1}$. Аналогічно 3 третього рівняння виражаємо $b_{2}$ через $a_{0}, a_{1}, a_{2}$. Продовжуючи цей процес $n-1$ раз всі $b_{k}$ при $k=1,2, \ldots, n-1$ будуть виражені через $a_{0}, a_{1}, \ldots, a_{k}$. При цьому $a_{0}, a_{1}, \ldots, a_{k}$ - довільні комплексні числа.

Зауважимо, що в теоремі 2.3 не стверджується лінійна незалежність векторів $e_{1}, e_{2}, e_{3}$.

Отже, ми довели навіть більше, що за умов теореми 2.3 вектор $e_{2}$ (або $e_{3}$ ) можна вибрати довільним, а тому знайдеться вектор $e_{3}$ (або $\left.e_{2}\right)$, який задовольняє характеристичне рівняння (1.3).

Теорему 2.3 можна узагальнити на випадок характеристичного рівняння вигляду:

$$
\sum_{\alpha, \beta, \gamma} C_{\alpha, \beta, \gamma} e_{1}^{\alpha} e_{2}^{\beta} e_{3}^{\gamma}=0
$$

тобто сума $\alpha+\beta+\gamma$ не рівна $N$. Ми також відмовилися від умови $e_{1}=1$.

Наслідок 2.4. $В$ кожній алгебрі виду $\mathbb{A}_{n}$ характеристичне рівняння (2.5) має розв'язки.

Доведення повністю аналогічне до доведення теореми 2.3, лише у системі (2.4) $R_{k}$ є поліномами степеня $\alpha+\beta+\gamma$, але не однорідними. 
Характеристичне рівняння (2.5) виникає тоді, коли ми розглядаємо лінійне диференціальне рівняння вигляду

$$
\sum_{\alpha, \beta, \gamma} C_{\alpha, \beta, \gamma} \frac{\partial^{N} U}{\partial x^{\alpha} \partial y^{\beta} \partial z^{\gamma}}=0, \quad C_{\alpha, \beta, \gamma} \in \mathbb{R} .
$$

$\mathrm{У}$ цьому випадку функція $\Phi(\zeta)=\exp \zeta$ при $\zeta=x e_{1}+y e_{2}+z e_{3}$ зі значеннями у будь-якій алгебрі виду $\mathbb{A}_{n} \in$ розв'язком рівняння (2.6), якщо вектори $e_{1}, e_{2}, e_{3}$ задовольняють рівняння (2.5). Вказаний підхід було реалізовано в роботі [10] для рівняння

$$
V_{t t t}(t, x)+\mu V_{t t}(t, x)-\nu V_{x x}(t, x)=0,
$$

при $\mu, \nu \in(0, \infty)$, яке використовується в одній моделі гідродинаміки.

\section{3. РОЗШИРЕННЯ АЛГЕБРИ ТА ЇХ ВЛАСТИВОСТІ}

Нехай $\widetilde{\mathbb{A}}_{n+1}$ - $(n+1)$-вимірна комутативна асоціативна алгебра 3 базисом $\left\{1, \widetilde{I}_{1}, \widetilde{I}_{2}, \ldots, \widetilde{I}_{n}\right\}$ виду $(2.1)$ :

$$
\forall r, s \in\{1,2, \ldots, n\} \quad \widetilde{I}_{r} \widetilde{I}_{s}=\sum_{k=\max \{r, s\}+1}^{n} \widetilde{\Upsilon}_{r, k}^{s} \widetilde{I}_{k} .
$$

Нехай, як і раніше, $\mathbb{A}_{n}-n$-вимірна комутативна асоціативна алгебра з базисом $\left\{1, I_{1}, I_{2}, \ldots, I_{n-1}\right\}$ і таблицею множення (2.1).

Означення 3.1. Алгебра $\widetilde{\mathbb{A}}_{n+1}$ називається розширенням алгебри $\mathbb{A}_{n}$, якщо справедливі рівності

$$
\begin{gathered}
\tilde{\Upsilon}_{r, k}^{s}=\Upsilon_{r, k}^{s} \\
\forall k \in\{2, \ldots, n-1\} \quad \forall r, s \in\{1,2, \ldots, k-1\} .
\end{gathered}
$$

Надалі розширення алгебри $\mathbb{A}_{n}$ позначатимемо через $\mathbb{E}\left(\mathbb{A}_{n}\right)$.

Зауваження 3.2. З умови (3.2) випливає, що $r, s=1,2, \ldots, n-2$. Тому для коректності означення необхідно, щоб $n \geq 3$. При $n=2$ за означенням покладаємо, що алгебра $\mathbb{A}_{3}(\alpha), \alpha \in \mathbb{C}$, з таблицею множення

\begin{tabular}{c||c|c|c}
$\cdot$ & 1 & $\widetilde{I}_{1}$ & $\widetilde{I}_{2}$ \\
\hline \hline 1 & 1 & $\widetilde{I}_{1}$ & $\widetilde{I}_{2}$ \\
\hline$\widetilde{I}_{1}$ & $\widetilde{I}_{1}$ & $\alpha \widetilde{I}_{2}$ & 0 \\
\hline$\widetilde{I}_{2}$ & $\widetilde{I}_{2}$ & 0 & 0 \\
\hline
\end{tabular}

є розширенням бігармонічної алгебри $\mathbb{B}$ (див., наприклад, [2]) з таблицею множення 


\begin{tabular}{c||c|c|}
$\cdot$ & 1 & $I_{1}$ \\
\hline \hline 1 & 1 & $I_{1}$ \\
\hline$I_{1}$ & $I_{1}$ & 0 \\
\hline
\end{tabular}

Зауважимо, що алгебра $\mathbb{A}_{3}(\alpha)$ при всіх $\alpha \in \mathbb{C}$ ізоморфна алгебрі $\mathbb{A}_{3}(1)$, моногенні функції в якій вивчались в роботі [5].

Зауваження 3.3. Іншими словами, рівність (3.1) означає, що якщо в таблиці множення виду $(2.2)$ алгебри $\mathbb{E}\left(\mathbb{A}_{n}\right)$ відкинути останній рядок і останній стовпчик і скрізь у таблиці множення елемент $\widetilde{I}_{n}$ замінити на нуль, то отримаємо таблицю множення алгебри $\mathbb{A}_{n}$.

Розглянемо приклади розширень.

Приклад 3.4. Кожна з наведених нижче алгебр є розширенням попередньої алгебри.

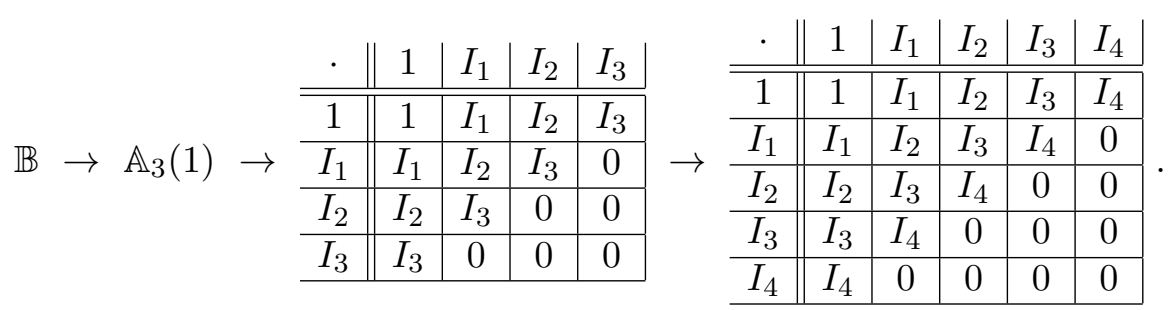

Можна говорити також про послідовність розширенъ. Очевидно, що наведені вище алгебри мають такі відповідні базиси: $\left\{1, I^{1}\right\}, I^{2}=0$; $\left\{1, I^{1}, I^{2}\right\}, I^{3}=0 ;\left\{1, I^{1}, I^{2}, I^{3}\right\}, I^{4}=0 ;\left\{1, I^{1}, I^{2}, I^{3}, I^{4}\right\}, I^{5}=0$. Для кожного натурального $n$ розглянемо алгебру з базисом $\left\{1, I^{1}, I^{2}, \ldots, I^{n}\right\}$, $I^{n+1}=0$. Очевидно, що $(n+1)$-ша алгебра є розширенням $n$-ї алгебри. Якщо $n$ пробігає всю множину натуральних чисел, то у такому випадку будемо казати, що маємо послідовність розширень.

Наведемо найпростіші властивості розширень.

$1^{\circ}$ Розширення алгебри $\mathbb{A}_{n}$ не єдине.

$2^{o}$ Довільна алгебра виду $\mathbb{A}_{n+1}$ є розширенням лише однієї алгебри.

Перейдемо до вивчення аналітичних властивостей в алгебрі $\mathbb{A}_{n}$ та в іiі розширенні $\mathbb{E}\left(\mathbb{A}_{n}\right)$.

Означення 3.5. На алгебрі $\mathbb{E}\left(\mathbb{A}_{n}\right)$ визначимо лінійний оператор

$$
\widetilde{P}: \mathbb{E}\left(\mathbb{A}_{n}\right) \mapsto \mathbb{A}_{n}
$$

рівностями

$$
\widetilde{P}(1)=1, \quad \widetilde{P}\left(\widetilde{I}_{k}\right)=I_{k} \quad \forall k=1,2, \ldots, n-1, \quad \widetilde{P}\left(\widetilde{I}_{n}\right)=0 .
$$


Тобто, для довільного $\tilde{a}:=a_{0}+\sum_{k=1}^{n} a_{k} \widetilde{I}_{k} \in \mathbb{E}\left(\mathbb{A}_{n}\right), a_{0}, a_{k} \in \mathbb{C}$ маємо $\widetilde{P}(\tilde{a})=a_{0}+\sum_{k=1}^{n-1} a_{k} I_{k} \in \mathbb{A}_{n}$.

Означення 3.6. На алгебрі $\mathbb{A}_{n}$ визначимо лінійний оператор

$$
P: \mathbb{A}_{n} \mapsto \mathbb{E}\left(\mathbb{A}_{n}\right)
$$

рівностями

$$
P(1)=1, \quad P\left(I_{k}\right)=\widetilde{I}_{k} \quad \forall k=1,2, \ldots, n-1 .
$$

Тобто, для довільного $a:=a_{0}+\sum_{k=1}^{n-1} a_{k} I_{k} \in \mathbb{A}_{n}, a_{0}, a_{k} \in \mathbb{C}$ маємо $P(a)=a_{0}+\sum_{k=1}^{n-1} a_{k} \widetilde{I}_{k} \in \mathbb{E}\left(\mathbb{A}_{n}\right)$.

Зауваження 3.7. Надалі у цій роботі для елемента $\tilde{a} \in \mathbb{E}\left(\mathbb{A}_{n}\right)$ і елемента $a \in \mathbb{A}_{n}$ використовуватимуться позначення, введені в означеннях 3.5 та 3.6.

Твердження 3.8. Оператор $\widetilde{P}$ узагальнено обернений відносно $P$.

Доведення проводиться шляхом безпосередньої перевірки рівності $P \widetilde{P} P=P$.

Теорема 3.9. Для довілъних $\tilde{a}, \tilde{b} \in \mathbb{E}\left(\mathbb{A}_{n}\right)$ справедлива рівність

$$
\widetilde{P}(\tilde{a} \tilde{b})=\widetilde{P}(\tilde{a}) \widetilde{P}(\tilde{b}) .
$$

Доведення. Розглянемо рівність

$$
\begin{aligned}
\widetilde{P}\left(\widetilde{I}_{s} \widetilde{I}_{r}\right) & =\widetilde{P}\left(\sum_{k=\max \{r, s\}+1}^{n} \Upsilon_{r, k}^{s} \widetilde{I}_{k}\right)=\sum_{k=\max \{r, s\}+1}^{n} \Upsilon_{r, k}^{s} \widetilde{P}\left(\widetilde{I}_{k}\right) \\
& =\sum_{k=\max \{r, s\}+1}^{n-1} \Upsilon_{r, k}^{s} I_{k}=I_{s} I_{r} .
\end{aligned}
$$

Тепер з урахуванням рівності (3.4), маємо

$$
\begin{aligned}
\widetilde{P}(\tilde{a} \tilde{b}) & =\widetilde{P}\left(\sum_{i, j=0}^{n} a_{i} b_{j} \widetilde{I}_{i} \widetilde{I}_{j}\right)=\sum_{i, j=0}^{n} a_{i} b_{j} \widetilde{P}\left(\widetilde{I}_{i} \widetilde{I}_{j}\right) \\
& =\sum_{i, j=0}^{n-1} a_{i} b_{j} I_{i} I_{j}=a b=\widetilde{P}(\tilde{a}) \widetilde{P}(\tilde{b}) .
\end{aligned}
$$


Наслідок 3.10. Для довільного $\tilde{a} \in \mathbb{E}\left(\mathbb{A}_{n}\right)$ такого, що $a_{0} \neq 0$ справедлива рівність

$$
\widetilde{P}\left(\tilde{a}^{-1}\right)=a^{-1}
$$

Доведення. Поклавши $\tilde{b}=\tilde{a}^{-1}$ в рівності (3.3) отримаємо

$$
\widetilde{P}\left(\tilde{a} \tilde{a}^{-1}\right)=\widetilde{P}(1)=1=\widetilde{P}(\tilde{a}) \widetilde{P}\left(\tilde{a}^{-1}\right)=a \widetilde{P}\left(\tilde{a}^{-1}\right) .
$$

Продемонструємо рівність (3.5)) на простому прикладі. Як зазначалося в зауваженні 3.2 , алгебра $\mathbb{A}_{3}(\alpha)$ є розширенням алгебри $\mathbb{B}$.

Приклад 3.11. В алгебрі $\mathbb{B}$ обернений елемент $a^{-1}$ має вигляд

$$
a^{-1}=\frac{1}{a_{0}}-\frac{a_{1}}{a_{0}^{2}} I_{1}, \quad a_{0} \neq 0 .
$$

$\mathrm{A}$ в алгебрі $\mathbb{A}_{3}(\alpha)$ обернений елемент $\tilde{a}^{-1}$ має такий вигляд

$$
\tilde{a}^{-1}=\frac{1}{a_{0}}-\frac{a_{1}}{a_{0}^{2}} \widetilde{I}_{1}+\left(\frac{a_{2}}{a_{0}^{2}}+\frac{a_{1}^{2}}{a_{0}^{3}} \alpha\right) \widetilde{I}_{2}, \quad a_{0} \neq 0 .
$$

На цих прикладах бачимо виконання рівності (3.5).

Означення 3.12. Для довільного $k \in \mathbb{N}$ та довільного $\tilde{a} \in \mathbb{E}\left(\mathbb{A}_{n}\right)$ визначимо $\tilde{a}^{-k}$ такою рівністю $\tilde{a}^{-k}:=\left(\tilde{a}^{-1}\right)^{k}$.

3 (3.3) випливає ще такий наслідок.

Наслідок 3.13. Для довільного иілого $k$ i довільного $\tilde{a} \in \mathbb{E}\left(\mathbb{A}_{n}\right)$ справедлива рівність

$$
\widetilde{P}\left(\tilde{a}^{k}\right)=(\widetilde{P}(\tilde{a}))^{k}
$$

Наступний наслідок випливає з рівностей (3.3) та (3.6).

Наслідок 3.14. Якщо вектори $\tilde{e}_{1}, \tilde{e}_{2}$, $\tilde{e}_{3}$ алгебри $\mathbb{E}\left(\mathbb{A}_{n}\right)$ задовольняють рівняння (2.5), то вектори $\widetilde{P}\left(\tilde{e}_{1}\right), \widetilde{P}\left(\tilde{e}_{2}\right), \widetilde{P}\left(\tilde{e}_{3}\right)$ алгебри $\mathbb{A}_{n}$ також задовольняють рівняння (2.5).

Приклад 3.15. Якщо вектори

$$
e_{1}=1, \quad e_{2}=a_{0}+a_{1} I_{1}+a_{2} I_{2}, \quad e_{3}=b_{0}+b_{1} I_{1}+b_{2} I_{2},
$$

$a_{k}, b_{k} \in \mathbb{C}$, алгебри $\mathbb{A}_{3}(\alpha)$ задовольняють рівняння $(2.5)$, то вектори

$$
e_{1}=1, \quad e_{2}=a_{0}+a_{1} I_{1}, \quad e_{3}=b_{0}+b_{1} I_{1}
$$

вже алгебри $\mathbb{B}$ також задовольняють рівняння (2.5).

Далі доведемо теорему, яка встановлює зв'язок між алгебрами виду $\mathbb{A}_{n}$ та їх розширеннями. 3 цією метою введемо таке означення. 
Означення 3.16. Нульовим розширенням алгебри виду $\mathbb{A}_{n}$ назвемо таке розширення $\mathbb{E}_{0}\left(\mathbb{A}_{n}\right)$ в якому

$$
\widetilde{\Upsilon}_{r, n+1}^{s}=0 \quad \forall r, s=1,2, \ldots, n .
$$

Іншими словами, таблиця множення нульового розширення $\mathbb{E}_{0}\left(\mathbb{A}_{n}\right)$ утворюється з таблиці множення алгебри $\mathbb{A}_{n}$ шляхом додавання $(n+1)$ го рядка і $(n+1)$-го стовпчика скрізь заповнених нулями крім тих клітинок де відбувається множення на одиницю алгебри.

Очевидно, що нульове розширення єдине. Наприклад, серед усіх розширень $\mathbb{A}_{3}(\alpha), \alpha \in \mathbb{C}$, алгебри $\mathbb{B}$ нульовим розширенням є лише алгебра $\mathbb{A}_{3}(0)$.

Якщо оператор $P$, який визначений в означенні 3.6 , приймає значення в нульовому розширенні $\mathbb{E}_{0}\left(\mathbb{A}_{n}\right)$, то подібно до оператора $\widetilde{P}$, він буде мультиплікативним. А саме, аналогічно до доведення теореми 3.9 доводиться наступне твердження.

Теорема 3.17. Для довільної алгебри виду $\mathbb{A}_{n}$ ma оператора

$$
P: \mathbb{A}_{n} \mapsto \mathbb{E}_{0}\left(\mathbb{A}_{n}\right)
$$

справедлива рівність

$$
P(a b)=P(a) P(b) \quad \forall a, b \in \mathbb{A}_{n} .
$$

Теорема 3.18. Якщо нулъові розширення $\mathbb{E}_{0}\left(\mathbb{V}_{n}\right)$ ma $\mathbb{E}_{0}\left(\mathbb{W}_{n}\right)$ алгебр виду (2.2) неізоморфні, то й алгебри $\mathbb{V}_{n} i \mathbb{W}_{n}$ також неізоморфні.

Доведення. Будемо доводити методом від супротивного. Припустимо, що алгебри $\mathbb{V}_{n}$ та $\mathbb{W}_{n}$ ізоморфні. Це означає, що існує таке лінійне взаємно однозначне відображення $\varphi: \mathbb{V}_{n} \rightarrow \mathbb{W}_{n}$, що для довільних $a, b \in \mathbb{V}_{n}$ справедлива рівність

$$
\varphi(a b)=\varphi(a) \varphi(b) .
$$

Нехай $\left\{I_{k}\right\}_{k=0}^{n-1}$ - базис алгебри $\mathbb{V}_{n},\left\{\widetilde{I}_{k}\right\}_{k=0}^{n}$ - базис алгебри $\mathbb{E}_{0}\left(\mathbb{V}_{n}\right)$, a $\left\{\widetilde{\rho}_{k}\right\}_{k=0}^{n}$ - базис алгебри $\mathbb{E}_{0}\left(\mathbb{W}_{n}\right)$. Визначимо відображення

$$
\psi: \mathbb{E}_{0}\left(\mathbb{V}_{n}\right) \rightarrow \mathbb{E}_{0}\left(\mathbb{W}_{n}\right)
$$

рівностями:

$$
\psi\left(\widetilde{I}_{k}\right)=P\left(\varphi\left(I_{k}\right)\right),(k=0,1, \ldots, n-1), \quad \psi\left(\widetilde{I}_{n}\right)=\widetilde{\rho}_{n} .
$$

Оскільки відображення $\varphi: \mathbb{V}_{n} \rightarrow \mathbb{W}_{n}$ та оператор $P: \mathbb{W}_{n} \rightarrow \mathbb{E}_{0}\left(\mathbb{W}_{n}\right)$ лінійні, то за визначенням (3.8) відображення $\psi: \mathbb{E}_{0}\left(\mathbb{V}_{n}\right) \rightarrow \mathbb{E}_{0}\left(\mathbb{W}_{n}\right)$ також лінійне. Це дає змогу за допомогою рівностей (3.8) визначити 
відображення $\psi$ для всіх $\tilde{a} \in \mathbb{E}_{0}\left(\mathbb{V}_{n}\right)$ :

$$
\psi(\tilde{a})=P(\varphi(a))+a_{n} \tilde{\rho}_{n} .
$$

Тепер для відображення $\psi: \mathbb{E}_{0}\left(\mathbb{V}_{n}\right) \rightarrow \mathbb{E}_{0}\left(\mathbb{W}_{n}\right)$ доведемо співвідношення

$$
\psi(\tilde{a} \tilde{b})=\psi(\tilde{a}) \psi(\tilde{b}), \quad \forall \tilde{a}, \tilde{b} \in \mathbb{E}_{0}\left(\mathbb{V}_{n}\right) .
$$

Беручи до уваги рівність (3.9), маємо співвідношення

$$
\psi(\tilde{a} \tilde{b})=P(\varphi(a b))+\left(a_{n} b_{0}+b_{n} a_{0}\right) \tilde{\rho}_{n} .
$$

Розглянемо добуток

$$
\begin{aligned}
\psi(\tilde{a}) \psi(\tilde{b}) & =\left(P(\varphi(a))+a_{n} \tilde{\rho}_{n}\right)\left(P(\varphi(b))+b_{n} \tilde{\rho}_{n}\right)= \\
& =P(\varphi(a)) P(\varphi(b))+\left(P(\varphi(a)) b_{n}+P(\varphi(b)) a_{n}\right) \tilde{\rho}_{n} .
\end{aligned}
$$

Оскільки оператор $P$ приймає значення в нульовому розширенні $\mathbb{E}_{0}\left(\mathbb{W}_{n}\right)$, то очевидними є рівності

$$
P(\varphi(a)) b_{n} \tilde{\rho}_{n}=a_{0} b_{n} \tilde{\rho}_{n}, \quad P(\varphi(b)) a_{n} \tilde{\rho}_{n}=b_{0} a_{n} \tilde{\rho}_{n} .
$$

Враховуючи співвідношення (3.7), (3.13), рівність (3.12) набуває вигляду

$$
\psi(\tilde{a}) \psi(\tilde{b})=P(\varphi(a b))+\left(a_{n} b_{0}+b_{n} a_{0}\right) \tilde{\rho}_{n} .
$$

Нарешті, наслідком рівностей (3.11), (3.14) є рівність (3.10).

Тепер покажемо, що відображення $\psi$ взаємно однозначне. За визначенням (3.8), матриця переходу від базису до базису має вигляд

$$
\widetilde{A}=\left(\begin{array}{cccc}
1 & 0 & \ldots & 0 \\
\alpha_{1,0} & \alpha_{1,1} & \ldots & \alpha_{1, n} \\
\vdots & \vdots & \ddots & \vdots \\
\alpha_{n-1,0} & \alpha_{n-1,1} & \ldots & \alpha_{n-1, n} \\
0 & 0 & \ldots & 1
\end{array}\right)
$$

Оскільки за припущенням алгебри $\mathbb{V}$ та $\mathbb{W}$ ізоморфні, то $\operatorname{det} A \neq 0$, де

$$
A=\left(\begin{array}{cccc}
1 & 0 & \ldots & 0 \\
\alpha_{1,0} & \alpha_{1,1} & \ldots & \alpha_{1, n-1} \\
\vdots & \vdots & \ddots & \vdots \\
\alpha_{n-1,0} & \alpha_{n-1,1} & \ldots & \alpha_{n-1, n-1}
\end{array}\right)
$$

Розкладаючи $\operatorname{det} \widetilde{A}$ за елементами останнього рядка, отримаємо

$$
\operatorname{det} \widetilde{A}=1 \cdot \operatorname{det} A \neq 0,
$$

а отже відображення $\psi$ є взаємно однозначним.

Таким чином, ми побудували лінійне взаємно однозначне відображення $\psi: \mathbb{E}_{0}\left(\mathbb{V}_{n}\right) \rightarrow \mathbb{E}_{0}\left(\mathbb{W}_{n}\right)$, яке задовольняє умову (3.10). Це означає, 
що алгебри $\mathbb{E}_{0}\left(\mathbb{V}_{n}\right)$ та $\mathbb{E}_{0}\left(\mathbb{W}_{n}\right)$ ізоморфні. Ми прийшли до суперечності з умовою теореми. Отже, алгебри $\mathbb{V}$ та $\mathbb{W}$ не є ізоморфними.

Зауваження 3.19. $З$ таблиць множення алгебр виду $\mathbb{A}_{n}$ для значень $n=2,3,4,5,6$ (про таблиці множення див. зауваження 5.3 в роботі [11]) видно, що нульові розширення неізоморфних $(n-1)$-вимірних алгебр виду $\mathbb{A}_{n-1}$ знову є неізоморфними. Теорема 3.18 є, в певному сенсі, оберненим результатом до вказаного факту, але для всіх натуральних $n \geq 2$.

\section{4. МОНОГЕННІ ФУНКЦІї НА РОЗШИРЕННЯХ АЛГЕБРИ $\mathbb{A}_{n}$}

Нехай

$$
e_{1}=1, \quad e_{2}=\sum_{r=0}^{n-1} a_{r} I_{r}, \quad e_{3}=\sum_{r=0}^{n-1} b_{r} I_{r}
$$

при $a_{r}, b_{r} \in \mathbb{C}$ - трійка векторів в алгебрі $\mathbb{A}_{n}$.

Нехай також $\zeta=x e_{1}+y e_{2}+z e_{3}$, де $x, y, z \in \mathbb{R}$. Комплексне число $\xi=x+y a_{0}+z b_{0}$ називається спектром точки $\zeta$. Виділимо в алгебрі $\mathbb{A}_{n}$ лінійну оболонку $E_{3}:=\left\{\zeta=x e_{1}+y e_{2}+z e_{3}: x, y, z \in \mathbb{R}\right\}$ породжену векторами $e_{1}, e_{2}, e_{3}$.

Далі істотним є припущення: $y a_{0}+z b_{0} \in \mathbb{C} \backslash \mathbb{R}$ при всіх дійсних $y, z$. Очевидно, що це має місце тоді і тільки тоді, коли хоча б одне з чисел $a_{0}$ чи $b_{0}$ належить $\mathbb{C} \backslash \mathbb{R}$. В теоремі 7.1 роботи [9] встановлено підклас рівнянь вигляду (1.1) для яких умова $y a_{0}+z b_{0} \in \mathbb{C} \backslash \mathbb{R}$ виконується при всіх дійсних $y, z$.

Області $\Omega$ простору $\mathbb{R}^{3}$ поставимо у відповідність область

$$
\Omega_{\zeta}:=\left\{\zeta=x e_{1}+y e_{2}+z e_{3}:(x, y, z) \in \Omega\right\}
$$

в $E_{3}$.

Неперервну функцію $\Phi: \Omega_{\zeta} \rightarrow \mathbb{A}_{n}$ називатимемо моногенною в області $\Omega_{\zeta} \subset E_{3}$, якщо $\Phi$ диференційовна за Гато в кожній точці цієї області, тобто якщо для кожного $\zeta \in \Omega_{\zeta}$ існує елемент $\Phi^{\prime}(\zeta)$ алгебри $\mathbb{A}_{n}$ такий, що виконується рівність

$$
\lim _{\varepsilon \rightarrow 0+0}(\Phi(\zeta+\varepsilon h)-\Phi(\zeta)) \varepsilon^{-1}=h \Phi^{\prime}(\zeta) \quad \forall h \in E_{3} .
$$

При цьому $\Phi^{\prime}(\zeta)$ називається похідною Гато функції $\Phi$ в точці $\zeta$.

Розглянемо розклад функції $\Phi: \Omega_{\zeta} \rightarrow \mathbb{A}_{n}$ за базисом $\left\{I_{k}\right\}_{k=0}^{n-1}$ :

$$
\Phi(\zeta)=\sum_{k=0}^{n-1} U_{k}(x, y, z) I_{k}
$$


У випадку, коли функції $U_{k}: \Omega \rightarrow \mathbb{C} \in \mathbb{R}$-диференційовними в області $\Omega$, тобто для довільного $(x, y, z) \in \Omega$

$$
\begin{aligned}
& U_{k}(x+\Delta x, y+\Delta y, z+\Delta z)-U_{k}(x, y, z)= \\
& =\frac{\partial U_{k}}{\partial x} \Delta x+\frac{\partial U_{k}}{\partial y} \Delta y+\frac{\partial U_{k}}{\partial z} \Delta z+o\left(\sqrt{(\Delta x)^{2}+(\Delta y)^{2}+(\Delta z)^{2}}\right),
\end{aligned}
$$

при $(\Delta x)^{2}+(\Delta y)^{2}+(\Delta z)^{2} \rightarrow 0$, функція $\Phi$ є моногенною в області $\Omega_{\zeta}$ тоді і тільки тоді, коли у кожній точці з $\Omega_{\zeta}$ виконуються умови:

$$
\frac{\partial \Phi}{\partial y}=\frac{\partial \Phi}{\partial x} e_{2}, \quad \frac{\partial \Phi}{\partial z}=\frac{\partial \Phi}{\partial x} e_{3} .
$$

Відмітимо, що розклад резольвенти має вигляд

$$
\left(t e_{1}-\zeta\right)^{-1}=\frac{1}{t-\xi}+\sum_{s=1}^{n-1} \sum_{k=2}^{s+1} \frac{Q_{k, s}}{(t-\xi)^{k}} I_{s}
$$

для всіх $t \in \mathbb{C}$ таких, що $t \neq \xi$, де $Q_{k, s}$ визначені наступними рекурентними співвідношеннями:

$$
Q_{2, s}:=T_{s}, \quad Q_{k, s}:=\sum_{r=k-2}^{s-1} Q_{k-1, r} B_{r, s}, \quad k=3,4, \ldots, s+1 .
$$

при

$$
T_{s}:=y a_{s}+z b_{s}, \quad B_{r, s}:=\sum_{k=1}^{s-1} T_{k} \Upsilon_{r, s}^{k}, \quad s=2,3, \ldots, n-1 .
$$

Із співвідношень (4.2) випливає, що точки $(x, y, z) \in \mathbb{R}^{3}$, які відповідають необоротним елементам $\zeta \in \mathbb{A}_{n}$, лежать на прямих

$$
L:\left\{\begin{array}{l}
x+y \operatorname{Re} a_{0}+z \operatorname{Re} b_{0}=0 \\
y \operatorname{Im} a_{0}+z \operatorname{Im} b_{0}=0
\end{array}\right.
$$

в просторі $\mathbb{R}^{3}$.

Нехай область $\Omega \subset \mathbb{R}^{3}$ опукла в напрямку прямої $L$. Позначимо

$$
D:=\left\{\xi=x+y a_{0}+z b_{0} \in \mathbb{C}: \zeta=x+y e_{2}+z e_{3} \in \Omega_{\zeta}\right\} .
$$

Теорема 4.1. [9] Нехай область $\Omega \subset \mathbb{R}^{3}$ опукла в напрямку прямої $L i$ нехай хоча б одне з чисел $a_{0}$ чи $b_{0}$ належсть $\mathbb{C} \backslash \mathbb{R}$. Тоді кожна моногенна бункція $\Phi: \Omega_{\zeta} \rightarrow \mathbb{A}_{n}$ подається у вигляді

$$
\Phi(\zeta)=\sum_{k=0}^{n-1} I_{k} \frac{1}{2 \pi i} \int_{\Gamma} F_{k}(t)(t-\zeta)^{-1} d t
$$


де $F_{k}$ - деяка голоморфна функиія в області $D$, a $\Gamma$-замкнена жорданова спрямлювана крива, яка лежить в області $D$ i охоплюе точку $\xi$.

Оскільки за умов теореми 4.1 кожна моногенна функція $\Phi: \Omega_{\zeta} \rightarrow \mathbb{A}_{n}$ продовжується до функції, моногенної в нескінченному циліндрі

$$
\Pi_{\zeta}:=\left\{\zeta \in E_{3}: \xi \in D\right\},
$$

то надалі будемо розглядати моногенні функції $\Phi$, визначені в областях виду $\Pi_{\zeta}$. Відмітимо, що циліндр $\Pi:=\left\{(x, y, z) \in \mathbb{R}^{3}: \zeta \in \Pi_{\zeta}\right\}$ паралельний прямій $L$.

В наступній теоремі встановлюється зв'язок між моногенними функціями в алгебрі виду $\mathbb{A}_{n}$ і моногенними функціями в $\dddot{1}$ довільному розширенні $\mathbb{E}\left(\mathbb{A}_{n}\right)$. Для формулювання результату введемо деякі позначення.

Нехай вектори $1, \tilde{e}_{2}, \tilde{e}_{3}$ алгебри $\mathbb{E}\left(\mathbb{A}_{n}\right)$ задовольняють характеристичне рівняння (1.3). На ці вектори натягнемо лінійний простір

$$
\widetilde{E}_{3}:=\left\{\widetilde{\zeta}=x+y \tilde{e}_{2}+z \tilde{e}_{3}: x, y, z \in \mathbb{R}\right\} .
$$

В алгебрі $\mathbb{A}_{n}$ будемо розглядати трійку $1, \widetilde{P}\left(\tilde{e}_{2}\right), \widetilde{P}\left(\tilde{e}_{3}\right)$ і лінійний простір

$$
\widetilde{P}\left(\widetilde{E}_{3}\right):=\left\{\zeta=x+y \widetilde{P}\left(\tilde{e}_{2}\right)+z \widetilde{P}\left(\tilde{e}_{3}\right): x, y, z \in \mathbb{R}\right\} .
$$

Теорема 4.2. Нехай вектори 1, $\tilde{e}_{2}, \tilde{e}_{3}$ виду (4.1) алгебри $\mathbb{E}\left(\mathbb{A}_{n}\right)$ задовольняють характеристичне рівняння (1.3) і нехай хоча б одне з чисел $a_{0}$ чи $b_{0}$ належить $\mathbb{C} \backslash \mathbb{R}$. Крім того, нехай функція $\widetilde{\Phi}: \widetilde{\Pi}_{\widetilde{\zeta}} \rightarrow \mathbb{E}\left(\mathbb{A}_{n}\right)$ змінної $\widetilde{\zeta}=x+$ уе $\tilde{e}_{2}+z \tilde{e}_{3}$ моногенна в деякому циліндрi $\widetilde{\Pi}_{\widetilde{\zeta}} \subset \widetilde{E}_{3}$. Тоді в алгебрі $\mathbb{A}_{n}$ трійка векторів $1, \widetilde{P}\left(\tilde{e}_{2}\right), \widetilde{P}\left(\tilde{e}_{3}\right)$ також задовольняе характеристичне рівняння (1.3), а функиіл $\Phi(\zeta):=\widetilde{P}(\widetilde{\Phi}(\widetilde{\zeta}))$ е моногенною в ииліндрі $\Pi_{\zeta}:=\left\{\zeta \in \widetilde{P}\left(\widetilde{E}_{3}\right): \widetilde{\zeta} \in \widetilde{\Pi}_{\widetilde{\zeta}}\right\}$ алгебри $\mathbb{A}_{n}$.

Доведення. Той факт, що вектори $1, \widetilde{P}\left(\tilde{e}_{2}\right), \widetilde{P}\left(\tilde{e}_{3}\right)$ задовольняють характеристичне рівняння (1.3) випливає з наслідку 3.14. Тепер зауважимо, що з тотожності (3.5) випливає тотожність

$$
(t-\zeta)^{-1}=\widetilde{P}\left((t-\widetilde{\zeta})^{-1}\right) \quad \forall t \in \mathbb{C}: t \neq x+y a_{0}+z b_{0} .
$$

За теоремою А функція $\widetilde{\Phi}$ подається у вигляді

$$
\widetilde{\Phi}(\widetilde{\zeta})=\sum_{k=0}^{n} \widetilde{I}_{k} \frac{1}{2 \pi i} \int_{\Gamma} \widetilde{F}_{k}(t)(t-\widetilde{\zeta})^{-1} d t .
$$


Подіємо на рівність (4.5) оператором $\widetilde{P}$. Враховуючи співвідношення (3.9) і (4.4), маємо

$$
\begin{aligned}
\widetilde{P}(\widetilde{\Phi}(\widetilde{\zeta})) & =\sum_{k=0}^{n} \widetilde{P}\left(\widetilde{I}_{k}\right) \frac{1}{2 \pi i} \int_{\Gamma} \widetilde{F}_{k}(t) \widetilde{P}\left((t-\widetilde{\zeta})^{-1}\right) d t= \\
& =\sum_{k=0}^{n-1} I_{k} \frac{1}{2 \pi i} \int_{\Gamma} \widetilde{F}_{k}(t)(t-\zeta)^{-1} d t .
\end{aligned}
$$

Відповідно до теореми А отримана функція $\widetilde{P}(\widetilde{\Phi}(\widetilde{\zeta}))$ є моногенною в циліндрі $\Pi_{\zeta}:=\left\{\zeta \in \widetilde{P}\left(\widetilde{E}_{3}\right): \widetilde{\zeta} \in \widetilde{\Pi}_{\widetilde{\zeta}}\right\}$.

Зауваження 4.3. Теорема 4.2 стверджує, що для вивчення моногенних функцій в алгебрах виду $\mathbb{A}_{n}$ при $n=2,3,4,5$ достатньо обмежитись дослідженням моногенних функцій у певних дев'яти алгебрах (які є нульовими розширеннями алгебр виду $\left.\mathbb{A}_{5}\right)$ виду $\mathbb{A}_{6}$.

Крім того, теорема 4.2 означає, що клас розв'язків рівняння (1.1) (i взагалі кажучи, рівняння (2.6)) у вигляді компонент моногенних функцій буде тим ширший чим більша розмірність алгебри виду $\mathbb{A}_{n}$. Або точніше: якщо комплекснозначний розв'язок $U(x, y, z)$ рівняння $(1.1)$ є деякою компонентою моногенної функції в деякій алгебрі $\mathbb{A}_{n}$ при $n<N$, то серед алгебр виду $\mathbb{A}_{N}$ існує алгебра $\mathbb{A}$ i існує моногенна функція $\Phi$ в $\mathbb{A}$ така, що $U(x, y, z)$ є деякою компонентою функції $\Phi$. При цьому $N$ може бути як завгодно великим.

Приклад 4.4. Розглянемо моногенні функції в алгебрі $\mathbb{B}$ і в її розширенні $\mathbb{A}_{3}(\alpha)$. В алгебрі $\mathbb{B}$ кожна моногенна функція подається у вигляді

$$
\Phi(\zeta)=F(\xi)+\left(\xi_{1} F^{\prime}(\xi)+F_{0}(\xi)\right) I_{1}, \quad \zeta=\xi+\xi_{1} I_{1}
$$

А з прикладу 3.11 і теореми А випливає, що кожна моногенна функція в алгебрі $\mathbb{A}_{3}(\alpha)$ подається у такому вигляді:

$$
\begin{aligned}
\widetilde{\Phi}(\widetilde{\zeta})=F(\xi)+\left(\xi_{1} F^{\prime}(\xi)+F_{0}(\xi)\right) \widetilde{I}_{1}+ \\
\quad+\left(\xi_{2} F^{\prime}(\xi)+\frac{\xi^{2} \alpha}{2} F^{\prime \prime}(\xi)+\xi_{1} F_{0}^{\prime}(\xi)+F_{1}(\xi)\right) \widetilde{I}_{2} \\
\widetilde{\zeta}=\xi+\xi_{1} \widetilde{I}_{1}+\xi_{2} \widetilde{I}_{2} .
\end{aligned}
$$

Якщо в $\widetilde{\Phi}(\widetilde{\zeta})$ покласти $\xi_{2}=0$, відкинути компоненту при $\widetilde{I}_{2}$ і $\widetilde{I}_{1}$ ототожнити з $I_{1}$, то з $\widetilde{\Phi}(\widetilde{\zeta})$ отримаємо $\Phi(\zeta)$, тобто моногенну функцію в звуженні $\mathbb{B}$. 
Зауваження 4.5. Теорема 4.2 залишається справедливою для моногенних функцій змінної $\zeta=x_{1} e_{1}+x_{2} e_{2}+\cdots+x_{d} e_{d}$ при $x_{1}, x_{2}, \ldots, x_{d} \in \mathbb{R}$, $e_{1}, e_{2}, \ldots, e_{d} \in \mathbb{E}\left(\mathbb{A}_{n}\right)$.

\section{ЛiтературА}

[1] E. Cartan. Les groupes bilinéares et les systèmes de nombres complexes. Annales de la faculté des sciences de Toulouse, 12(1):1-64, 1898.

[2] S. V. Grishchuk, S. A. Plaksa. Monogenic functions in a biharmonic algebra. Ukr. Math. J., 61(12):1865-1876, 2009.

[3] P. W. Ketchum. Analytic functions of hypercomplex variables. Trans. Amer. Math. Soc., 30(2):641-667, 1928.

[4] I. P. Mel'nichenko. The representation of harmonic mappings by monogenic functions. Ukr. Math. J., 27(5):599-505, 1975.

[5] S. A. Plaksa, V. S. Shpakovskii. Constructive description of monogenic functions in a harmonic algebra of the third rank. Ukr. Math. J., 62(8):1251-1266, 2011.

[6] A. Pogorui, R. M. Rodriguez-Dagnino, M. Shapiro. Solutions for pdes with constant coefficients and derivability of functions ranged in commutative algebras. Math. Meth. Appl. Sci., 37(17):2799-2810, 2014.

[7] M. N. Roşculeţ. Algebre infinite asociate la ecuaţii cu derivate parţiale, omogene, cu coeficienţi constanţi de ordin oarecare. Studii şi Cercetări Matematice, 6(3-4):567-643, 1955.

[8] M. N. Roşculeţ. Algebre infinite, comutative, asociate la sisteme de ecuaţii cu derivate parţiale. Studii şi Cercetări Matematice, 7(3-4):321-371, 1956.

[9] V. S. Shpakivskyi. Constructive description of monogenic functions in a finitedimensional commutative associative algebra. Adv. Pure Appl. Math., 7(1):63-75, 2016.

[10] В. Шпаковский. Гиперкомплексные функции и точные решения одного уравнения гидродинамики. Зб. праць Ін-ту математики НАН України, 14(1):262-274, 2017.

[11] В. С. Шпаківський. Про моногенні функції, визначені в різних комутативних алгебрах. Прийнято до друку в Укр. мат. вісник, arXiv:1803.03938v1.

Надійшла до редакції 9 квітня 2018, прийнята до друку 20 серпня 2018.

\section{B. С. Шпаківський}

ІНСТИТУТ МАТЕМАТИКИ НАН УКРАЇнИ

Email: shpakivskyi86@gmail.com 